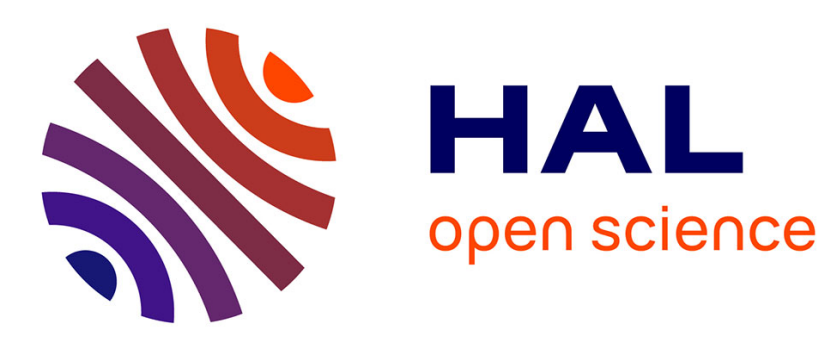

\title{
Un pugile ai piedi dell'Olimpo: un nuovo epigramma da Azoros (Tessaglia)
}

Eleonora Santin, Athanasios Tziaphalias

\section{To cite this version:}

Eleonora Santin, Athanasios Tziaphalias. Un pugile ai piedi dell'Olimpo: un nuovo epigramma da Azoros (Tessaglia). Revue de philologie, de littérature et d'histoire anciennes , 2010, 2008 (2), pp.377384. hal-01674730

\author{
HAL Id: hal-01674730 \\ https://hal.science/hal-01674730
}

Submitted on 28 Jan 2020

HAL is a multi-disciplinary open access archive for the deposit and dissemination of scientific research documents, whether they are published or not. The documents may come from teaching and research institutions in France or abroad, or from public or private research centers.
L'archive ouverte pluridisciplinaire HAL, est destinée au dépôt et à la diffusion de documents scientifiques de niveau recherche, publiés ou non, émanant des établissements d'enseignement et de recherche français ou étrangers, des laboratoires publics ou privés. 


\section{R E V UE}

DE

P H I L O L O G I E DE LITTÉRATURE ET D'HISTOIRE ANCIENNES

TOME 82

2008

FASCICULE 2 


\title{
REVUE
}

\author{
DE
}

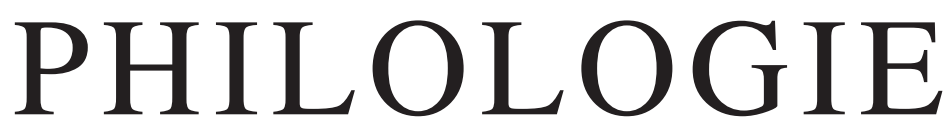

DE LITTÉRATURE ET D'HISTOIRE ANCIENNES

\section{TROISIÈME SÉRIE}

PUBLIÉE SOUS LA DIRECTION DE

\author{
M. CASEVITZ \\ PROFESSEUR ÉMÉRITE \\ À L'UNIVERSITÉ \\ DE PARIS OUEST NANTERRE
}

ET

\author{
Ph. MOREAU \\ PROFESSEUR À \\ L'UNIVERSITÉ \\ DE PARIS EST CRÉTEIL
}

\section{ANNÉE ET TOME LXXXII \\ FASC. 2 \\ (144 ${ }^{\mathrm{e}}$ de la collection)}

PARIS

KLINCKSIECK 
Retrouvez les sommaires de la Revue de philologie et les nouveautés Klincksieck sur www.klincksieck.com

ISBN 978-2-252-03719-5

(C) Klincksieck, 2011 


\section{UN PUGILE AI PIEDI DELL'OLIMPO : UN NUOVO EPIGRAMMA DA AZOROS (TESSAGLIA)}

I monti e le pianure della Tessaglia furono, ben si sa, una grande palestra d'eroi, teatro delle imprese di Eracle, Giasone, Admeto, Peleo ed Achille. Non è forse altrettanto noto che in più occasioni gli atleti tessali emularono i loro mitici predecessori trionfando alle Olimpiadi o in altre gare panelleniche ${ }^{1}$. Essi diedero così ai poeti ampia materia per elaborare un canto di vittoria che fungesse da cassa di risonanza per la fama delle loro nobili famiglie e di quelle dei loro patroni ${ }^{2}$.

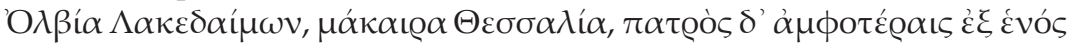

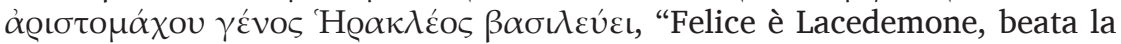
Tessaglia, su entrambe regna la discendenza di uno solo padre, Eracle, il migliore in battaglia", è questo l'altisonante incipit della X Pitica che Pindaro ventenne compose, su richiesta del tagos alevade Thorax di Larissa, per celebrare la vittoria nel diaulos dei ragazzi della $22^{\text {a }}$ Pitiade (498 a. C.) del nobile Hippokleas di Pelinna, due volte olympionikes, figlio di Phrikias, a sua volta pluricampione olimpico nell'hoplitodromos. Bacchilide scrisse quella che è ora l'Ode XVI in onore di Aristotele di Larissa e del suo duplice successo ai

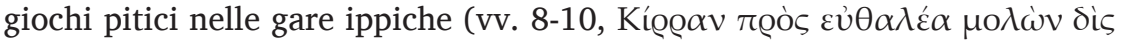

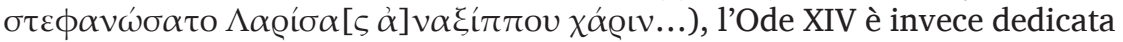
al tessalo Kleoptolemos vincitore nella corsa col carro ai Petraia, manifestazione locale in onore di Poseidone Petraios organizzata a partire dall'inizio del V sec. a. C. ${ }^{3}$. Quanto a Simonide, di cui è noto il contatto con l'ambiente

1. Il tema sport e competizioni agonistiche in Tessaglia è stato al centro di un'esposizione temporanea allestita presso il Museo di Volos in occasione dei giochi olimpici del 2004, si rinvia al catalogo della mostra per una più ampia e dettagliata panoramica sulle questioni accennate in questa breve introduzione e per una lista completa dei vincitori tessali nelle gare olimpiche (redatta sulla base di L. Moretti, Olympionikai, $i$ vincitori negli antichi agoni olimpici, Atti della Accademia Nazionale dei Lincei, s. VIII Memorie, vol. VIII, 2, Roma, 1959, p. 53-198), vd. Ministry of Culture, Volos Archaeological Museum, Games and Sports in ancient Thessaly, Athens, 2004.

2. Sul ruolo e la partecipazione delle famiglie dominanti tessale negli agoni panellenici tra il VI e il IV sec. a. C., vd. M. Stamatopoulou, Thessalian Aristocracy and Society in the Age of Epinikian in S. Hornblower, C. Morgan (eds.), Pindar's Poetry, Patrons and Festivals, Oxford, 2007, p. 309341. Nella ricca rassegna di fonti archeologiche citate dall'autrice si segnala in particolare l'altère votivo in bronzo con epigrafe metrica entrato nel 1993 nella collezione di M.G. Ortiz, dedicato ad Apollo Hekabolos per ricordare una vittoria nel pentathlon : l'oggetto è stato certamente offerto da un atleta tessalo nel secondo quarto del V sec. a. C., cf. D. Knoepfler, Haltère de bronze dédié à Apollon Hékabolos dans la collection G. Ortiz (Genève), CRAI 138, N. 2, (1994), p. 337-379.

3. Cf. Stamatopoulou, cit., p. 333 e Games and Sports, cit., p. 57-58. 
tessalo in seguito al suo soggiorno presso Skopas il Giovane di Krannon ${ }^{4}$, sappiamo che il frammento PMG 511 (= P.Oxy 2431) faceva parte di un componimento probabilmente scritto per un tessalo vincitore a Delfi.

Pausania $^{5}$ descrisse nelle vesti di nuovo Eracle Poulydamas figlio di Nikias di Skotoussa ${ }^{6}$, vittorioso nel pankration dell'anno 408 a. C. Le sue 'fatiche', tra cui l'uccisione di un leone a mani nude sul monte Olimpo, erano in parte scolpite nei rilievi e in parte illustrate in un'iscrizione sulla base della statua a lui dedicata vari anni dopo la vittoria ad Olimpia dai suoi compatrioti, opera dello scultore Lisippo. Periodonikai, attivi all'inizio del V sec. a. C., furono i fratelli Telemachos e Agias figli di Aknonios di Pharsalos, uno nella lotta, l'altro nel pankration ${ }^{7}$. Agias, fu il primo fra i suoi concittadini, a guadagnare la vittoria olimpica nella sua specialità, come ricorda non solo l'epigramma inciso sul celebre monumento delfico di Daochos ${ }^{8}$, ma anche la frammentaria iscrizione presente sulla base della statua a lui innalzata a Pharsalos recante, anche in questo caso, la firma di Lisippo ${ }^{9}$.

La celebrazione degli atleti in vita assunse dunque dapprima la forma dell'epinicio in metro lirico per poi confluire, a partire dal IV secolo a. C., in elegie ed epigrammi forgiati in buona parte sull'esempio delle numerose iscrizioni agonistiche metriche che accompagnavano statue e doni votivi ${ }^{10}$. L'elogio in morte è invece suggellato da epitaffi con un evidente debito strutturale e tematico verso tali espressioni letterarie ed epigrafiche di encomio della vis atletica. La memoria dei campioni dello stadio e dell'arena viene a fissarsi definitivamente nei versi incisi sul loro monumento funerario : è

4. Sulla permanenza di Simonide presso Skopas figlio di Creonte cf. Pl. Prot, 339a, M. Sordi, La lega tessala fino ad Alessandro Magno, Roma, 1958, p. 61-62 e B. Helly, L'État thessalien, Aleuas le Roux, les tétrades et les tagoi, Lyon, 1995, p. 108-109.

5. Cf. Paus., VI, 5, 4-6.

6. Vd. K. Scherling, RE, s.u. Poulydamas, n. 4 (1952), L. Moretti, art. cit., p. 110, n. 348 ; B. Helly, Des lions dans l'Olympe, REA 70 (1968), p. 278-285. Sulla statua olimpica cf. G. Treu, Olympia III, p. 209 sq., tav. LV, 1-3, Stamatopoulou, cit., p. 339-340.

7. Cf. R. Knab, Die Periodoniken, repr. Chicago, 1980, n. 11/12, Moretti, cit. n. 190 e 192, J. Ebert, cit. infra, n. 43-45. Il terzo dei figli di Aknonios, Agelaos, vinse a Delfi nello stadion dei ragazzi.

8. FD III 4, 460, 337-332 a. C., vd. A. Jacquemin, D. Laroche, Le monument de Daochos ou le trésor de Thessaliens, BCH 125 (2001), p. 305-332.

9. IG IX 2249 = Decourt, IThess. 57.

10. Sulle iscrizioni agonistiche opera di riferimento resta naturalmente L. Moretti, Iscrizioni agonistiche greche, Roma 1953, sugli epigrammi per vincitori negli agoni fondamentale è J. Ebert, Griechische Epigramme auf Sieger an gymnischen und hyppischen Agonen, Berlin 1972, per i pugili si confronteranno i n. 11, 14, 16, 21, 30, 31, 37, 55, 61, 62, 67. 70. Un quadro d'insieme sulle composizioni per atleti di età ellenistica, con particolare riferimento all'encomio dei pugili, è tracciato da S. Barbantani, Epinici in distici di età ellenistica. Una composizione sul pugilato ? Nota su PSchubart 13 (OBerol 9303), PapLup 9 (2000), p. 65-73. Per la stretta relazione tra epinicio in metro lirico ed iscrizioni agonistiche si veda P. Bernardini, Epinici e iscrizioni agonistiche : un percorso da ricostruire, in Cannatà Fera-Grandolini ed., Poesia e religione in Grecia. Studi in onore di G.Aurelio Privitera, Napoli, 2000, p. 29-41 
questo l'ultimo tributo levato alla gloria ed alla memoria di uomini stimati quali eroi della patria al pari dei caduti in battaglia ${ }^{11}$.

Un nuovo piccolo contributo alla storia di questa categoria emerge dalle poche lettere ancora visibili su una stele rinvenuta nel sito dell'antica Azoros, una delle città che componevano la cosiddetta Tripolis, zona del territorio tessalo situata ai piedi dell'Olimpo nell'alta valle del fiume Titaresos ${ }^{12}$. L'iscrizione, così ricostruita, ci restituisce un semplice distico elegiaco in memoria di un giovane pugile perito in ignote circostanze dopo aver calcato le orme di Admeto e del Lapite Mospos ${ }^{13}$ con una vittoria nella categoria dei ragazzi in un non menzionato contesto agonistico.

\section{GHW $6148^{14}$}

Luogo di ritrovamento : Azoros (Vouvala). Città antica : Azoros.

Monumento : grande stele calcarea con coronamento ogivale rotta sul lato sinistro per circa un terzo della sua larghezza. L'iscrizione attualmente

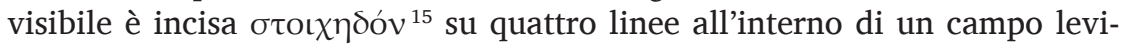
gato.

Dimensioni : h 1,00 (coronamento h 0,25 ) ; $1.0,39$; sp. 0,14-0,15. Lettere : h 0,012-0,015. Interlinea : 0,008-0,001.

Luogo di conservazione : Azoros (demarchia), inv. n. Az./40.

Datazione : l'epigramma appartiene ad una serie di iscrizioni metriche del sito di Azoros, ancora inedite, collocabili nel IV sec. a. C. Si propende per una datazione piuttosto alta, verso l'inizio del secolo, per un insieme di motivazioni : la forma dei caratteri, la disposizione stoichedica e la presenza di un genitivo in -ō attestato in iscrizioni metriche e in prosa di V/IV a. C.

$$
\begin{aligned}
& \text { [.....] Е П A I } \Sigma \text { K E I T A [...] } \\
& \text { [.....] ОО.ПО Т Е П Г М [. .] }
\end{aligned}
$$

11. La poesia funeraria per atleti e guerrieri è il tema centrale della raccolta di A. Stecher, Inschriftliche Grabgedichte auf Krieger und Atleten : eine Studie zu griechischen Wertprädikationen, Innsbruck, 1981.

12. Le altre città sono, come noto, Doliche e Phytion, vd. G. Lucas, Les cités antiques de la haute vallée du Titarèse, Lyon, 1997, per il sito e la documentazione epigrafica di Azoros, cf. p. $157 s q$.

13. Il cui scontro era rappresentato sull'arca di Cipselo descritta da Pausania, V, 17, 10.

14. Fotografie e calco dell'iscrizione si trovano presso gli archivi della Maison de l'Orient et de la Méditerranée Jean Pouilloux di Lione, Laboratorio HiSoMA (Histoire et Sources des Mondes Antiques, UMR 5189). Nella banca dati GHW (Graeve-Helly-Wolters) l'iscrizione è la n. 6148, il calco TH03126.

15. Una lista di iscrizioni metriche tessaliche incise con la medesima tecnica, tutte di V secolo, è in E. Santin, Nuova lettura dell'epigramma funerario per Diokleas, ZPE 166 (2008), nota 4, p. 73, si aggiungano le dediche votive CEG 341 e $C E G$ 342. Per un catalogo provvisorio di

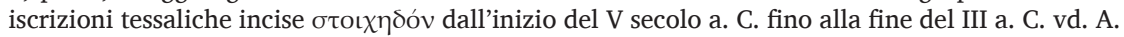

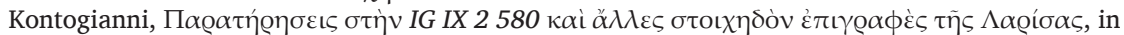

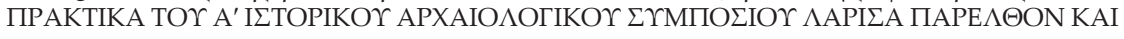
ME $\Lambda$ ON, Larissa, 1985, p. 111-132. 


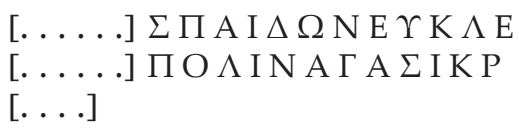

Note

La frattura sul lato sinistro ha determinato una lacuna di cinque caratteri nella prima e nella seconda linea e di sei nella terza e quarta.

1. 1 Prima di epsilon si distingue la metà di una lettera triangolare, molto probabilmente un delta. Dopo ЕП la pietra ha subito una scalfittura superficiale che ha compromesso la lettura di una lettera sia nella prima che nella seconda linea. Sembra comunque di vedere un segno triangolare, un alpha molto aperto, si restituisce pertanto $\pi \alpha$ ĩ $\varsigma$. Alla fine della linea lacuna di tre lettere dovuta al danneggiamento della pietra.

11. 1-2 Tra la prima e la seconda linea doveva trovarsi il nome del padre di Agasikrates al genitivo, si propone [....... $] \overline{\mathrm{o}}$, ő[ $\varsigma]$ dove $\overline{\mathrm{o}}=$ ov (vd. commento).

1. 3 L'integrazione $[v \iota \kappa \eta ́ \sigma \alpha] \varsigma$ è quella che meglio d'ogni altra si adatta alla menzione di una vittoria del defunto nelle gare di pugilato dei $\pi \alpha \tilde{i} \delta \varepsilon \varsigma$ (vd. commento).

1. 4 L'avverbio $\pi$ ó $\varepsilon^{16}$ richiede un verbo al passato, per questo $\varepsilon \dot{\jmath} \kappa \lambda \varepsilon[i ́ \sigma \varepsilon v]$, l'assenza d'aumento non pone particolari problemi in un'iscrizione metrica dove l'omissione può essere dovuta proprio a ragioni di convenienza prosodica ${ }^{17}$.

1. 5 L'esistenza di una quinta linea va necessariamente supposta per poter completare il nome del defunto.

Qui giace il figlio di [...] che un tempo, avendo vinto la gara di pugilato dei ragazzi, ricoprì di gloria la città, Agasikrates.

La presentazione metrica è la seguente :

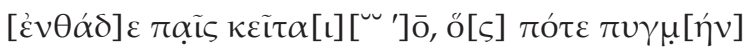

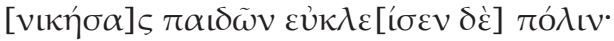

А $\gamma \alpha \sigma \iota{ }^{\prime}[\alpha ́ \tau \eta \varsigma]$

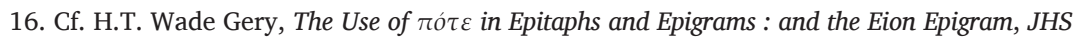
53 (1934), p. 71-82.

17. Vd. K. Mickey, Studies in the Greek Dialects and the Language of Greek Verse Inscriptions, Oxford, 1981, Ch. 2, p. 26, Ch. 3, p. 36 e Ch. 5 V. 1, p. 246. 


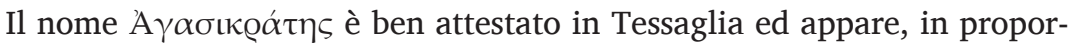
zione minore ma non trascurabile, anche nella forma dialettale A $\gamma \alpha \sigma \iota \kappa \varrho \alpha ́ \tau \varepsilon ı \varsigma$ (vd. Fraser-Matthews, LGPN IIIB, s.u.).

Nella lacuna tra la prima e la seconda linea si è scelto di restituire [ ${ }^{u}$ '] $\overline{\mathrm{o}}, \mathrm{o}[\varsigma] \pi \hat{\tau} \tau \varepsilon \pi v \gamma \mu[\eta \dot{v} v]$. Tale struttura sintattica, con una proposizione relativa posta immediatamente dopo le usuali formule "qui giace il tale figlio del tale" oppure "questo è il monumento di", oltre ad essere generalmente diffusa nella poesia esametrica sia letteraria che epigrafica, è ricorrente in iscrizioni di età arcaico-classica in Tessaglia ${ }^{18}$ come pure nelle epigrafi metriche dedicate ad atleti ${ }^{19}$. Per la notazione di $|o|$ lungo sono utilizzati omicron per il suono lungo chiuso e omega per quello lungo aperto. L'uso di $\bar{o}$ e $\omega$ per la resa della vocale lunga chiusa e lunga aperta trova riscontro sia in area eolica sia, in un contesto geografico più ampio, in diversi altri epigrammi di epoca arcaico-classica, esso denota una lunga fase di passaggio che si protrae fino alla prima metà del IV secolo a. C. e all'introduzione stabile della notazione ov ${ }^{20}$.

Quasi certamente ci sarà stata nel nostro epigramma una distinzione tra $\varepsilon$ ed $\eta$, anche se non possiamo affermarlo con assoluta certezza, dato che tutti gli eta sono in lacuna ${ }^{21}$.

Una desinenza -ō per il nome del padre di Agasikrates è perfettamente ammissibile : un genitivo così espresso ${ }^{22}$ risulta attestato nella regione ${ }^{23}$. Esso appare ad esempio nella stessa sede metrica e nello stesso giro sintattico in un epigramma rinvenuto sulle pendici del monte Pelio risalente al V secolo a. C. (Hansen, CEG 123) :

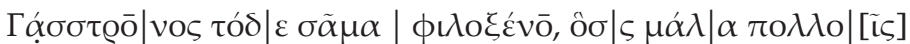

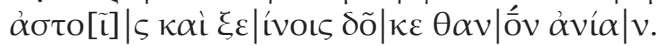

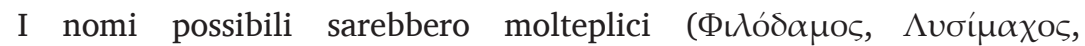
$\Pi v \theta o ́ \delta \omega \varrho o \varsigma$, etc.) e non sussistono ragioni per prediligerne uno in particolare.

18. Si vedano ad es. gli epigrammi per Pyrhiadas (Hansen, CEG 118), Gastron (Hansen, CEG 123, cf. il suo gemello Peek, GV 321, Thisbe V a. C.), Thibron (Hansen, CEG 641), Sosikrates (A. Tziaphalias, $A D 35$ B1 (1980) [1988], p. 295) e quello per un altro atleta tessalo Menon (Hansen, CEG 643).

19. Come ad esempio nell'epigramma per Milone di Crotone figlio di Diotimos, famoso lottatore più volte vincitore ai giochi olimpici, vd. Ebert, n. $61.1=$ AP XVI 24, attribuito a Simonide

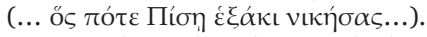

20. Cf. W. Blümel, Die aiolischen Dialekte, Göttingen, 1982, p. 32, cf. Hansen CEG 82, 83, 93, $162,478,360,664,665$.

21. In altre iscrizioni di Azoros del IV secolo la distinzione grafica lunga/breve non è sempre netta : in un epigramma inedito databile al IV sec. a. C. (GHW 6138) abbiamo $\varepsilon / \eta, o / o v, ~ m \alpha ~ o$

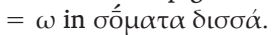

22. Vd. W. Blümel, op. cit. § 44 e $\S 252$, Mickey, cit. Ch. 2, p. 26 e nota 66.

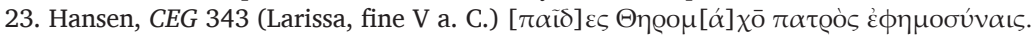




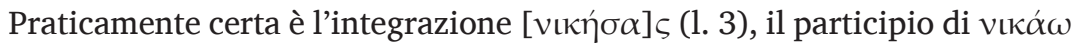
è conforme all'uso formulare e si colloca in una sede metrica usuale ${ }^{24}$. Il genitivo $(\pi \alpha \mathrm{\iota} \delta \tilde{\omega} v / \hat{\alpha} v \delta \varrho \tilde{\omega} v)$ per esprimere la categoria è nelle iscrizioni meno comune dell'accusativo $\pi \alpha \tilde{i} \delta \alpha \varsigma / \alpha \ddot{\alpha} \nu \delta \alpha \varsigma$, ma lo troviamo impiegato in un considerevole numero di casi ${ }^{25}$, ad Aphrodisias, in Caria, ad esempio, è l'unica formulazione ad oggi attestata ${ }^{26}$.

Agasikrates doveva essere un giovane nel fiore degli anni, il poeta lo designa unicamente come "figlio del tale" ( $\pi \alpha \underline{i} \varsigma$ [....... ō) e non come marito o padre ; la menzionata vittoria nelle gare di pugilato dei $\pi \alpha \tilde{i} \delta \varepsilon \varsigma$, alle quali deve aver partecipato in un'età grossomodo compresa tra i diciassette e i diciannove anni, non poteva essere dunque troppo remota ${ }^{27}$. Non viene dichiarato il contesto in cui tale successo fu riportato, come invece accade di norma negli epinici di natura letteraria e nelle iscrizioni metriche onorarie e anatematiche relative ad atleti. Molto probabilmente la vittoria fu conseguita nell'ambito di gare internazionali, nelle quali la partecipazione di atleti tessali è ben attestata almeno fino all'inizio del IV secolo a. C., piuttosto che nel corso di competizioni locali ${ }^{28}$. Il riferimento alla gloria procurata alla patria dai trionfi di un atleta nei giochi panellenici è infatti una costante nell'encomio dei vincitori. I possibili raffronti sono numerosi, mi limito pertanto a citare un paio tra i più evidenti casi di corrispondenza nelle scelte lessicali degli autori di questo ed altri epigrammi : Ebert $41=$ IThesp 208, Tespie, metà del IV a. C. per Aischylos vincitore olimpico nella

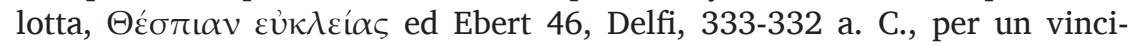

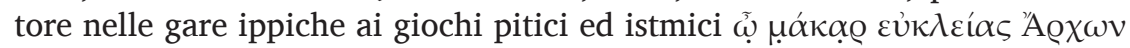

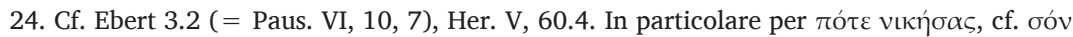

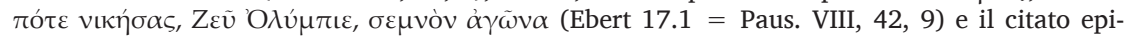

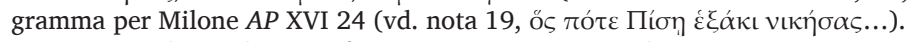

25. Si vedano ad es. $I G \mathrm{II}^{2}$ 2326, B.D. Meritt, Corinth VIII, 1 15, IG XI 2, 205, McCabe, Tralles, n. 69, 73 e 75, McCabe, Chios, 57.

26. IAph (online corpus, http://insaph.kcl.ac.uk/iaph2007/index.html, cons. in data 20 febbraio 2010), n. 11.60, 11.223 e 12.35 .

27. Per l'elogio delle qualità atletiche negli epigrammi funerari per adolescenti e giovani uomini vd. A.-M. Vérilhac, Paides Aoroi, vol. II, Athènes, 1982, p. 51-58. In particolare, per i poemi destinati a giovani pugili e per le questioni riguardanti l'età dei combattenti vd. p. 54-55.

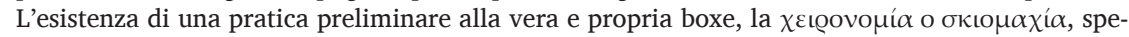
cialità basata solo sull'abilità nell'effettuare finte e parate, abbassa notevolmente l'età dei ragazzi ricordati nelle iscrizioni come pugili (cf. Vérilhac, n. 68, per un bambino di 9 anni, Roma, II/ III d. C.). Pare tuttavia certo che i partecipanti agli agoni ufficiali nella categoria dei $\pi \alpha \tilde{i} \delta \varepsilon \varsigma$ praticassero il pugilato normale, una forma di combattimento molto violenta per la quale erano necessari una corporatura ed un peso raggiungibili solo nella piena adolescenza. Sulla pratica del pugilato nell'antica Grecia la letteratura è molto ricca, si vedano M.B. Poliakoff, Combats Sports in the Ancient World, Ch. V, New Haven, 1987, p. 68-88, W.E. Sweet, Sport and Recreation in Ancient Greece. A Sourcebook with Translations, Oxford, 1987, p. 68-80, A. Papalas, The development of Greek boxing, The Ancient World IX (1984) 3-4, p. 67-76.

28. Sulle quali, va ribadito, siamo poveri di informazioni per il periodo precedente l'istituzione degli Eleutheria celebrati a partire dal 196 a. C per festeggiare la 'liberazione' dalla dominazione macedone. Cf. Stamatopoulou, art. cit., p. 333-337. 


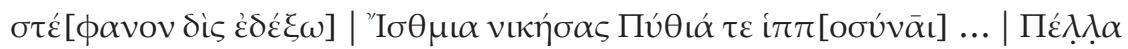

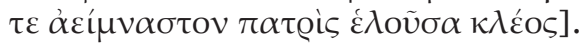

Non sono altresì specificate le circostanze del decesso. Anche se il combattimento a livello agonistico è non di rado una fase preparatoria o concomitante agli esordi della carriera militare ${ }^{29}$, è improbabile che il giovane sia caduto in guerra in quanto una simile ragione sarebbe stata senza dubbio espressa. In effetti, dovendo ridurre alla brevità della forma lapidaria la vita di Agasikrates, il poeta ha promosso al rango di evento saliente, pur lasciando in ombra l'occasione specifica, solo la vittoria che lo innalzò ai pubblici onori ${ }^{30}$. Più ampio respiro ha invece l'epigramma per Menon, un

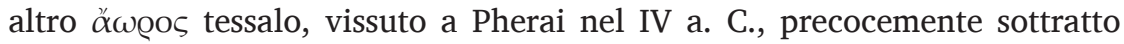
ai successi di una brillante carriera atletica (vd. Hansen, CEG 643, 350-300 a. C.). Si noterà tuttavia come lo stesso ordito tematico si intrecci su una simile trama sintattica.

$\mathrm{E} \lambda \lambda \dot{\alpha} \varsigma$

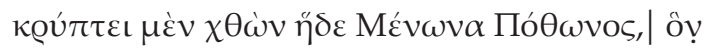

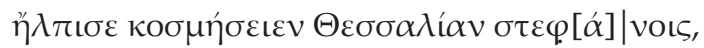

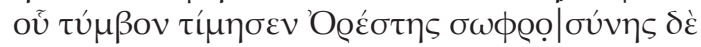
4

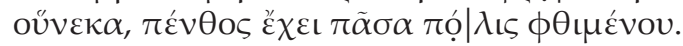

Questa terra ricopre Menon figlio di Pothon, che l'Ellade sperò potesse fregiare di vittorie la Tessaglia, fu invece Oreste ad onorarne la tomba per la sua saggezza, l'intera città lo rimpiange ora che è morto.

In conclusione, il distico per Agasikrates ci conferma come la composizione della sequenza di versi destinata al monumento sepolcrale di un atleta sia principalmente ispirata ai temi e alle formule dell'encomio in vita. Semplice ed essenziale, esso si colloca alle origini di una serie epigrafica che si evolve nel tempo ${ }^{31}$ gonfiata da una retorica incline a calcare sempre più i

29. Vd. Ebert 70 = Peek, GV 1004 ed Ebert 50 per un ex-pugile e un ex-lottatore entrambi morti in battaglia.

30. Nel giro di due soli versi l'epigramma soddisfa i punti fondamentali intorno ai quali si sviluppano le composizioni del genere epigrammatico agonistico : la menzione del padre, il numero e la tipologia delle vittorie, il tema della gloria patria. Formulazioni più estese di iscrizioni metriche per pugili incentrate sui medesimi elementi portanti sono nello stesso periodo Hansen, CEG 844 (Thasos, 400-350 a. C.) per Timoxenos, pugile più e più volte vincitore ai giochi panellenici e CEG 801 (Delfi, 380-370 a. C.) per il figlio di Taureas campione a Delfi nel pugilato dei $\pi \alpha \tilde{i} \delta \varepsilon \varsigma$.

31. Gli esempi sarebbero numerosi, per un excursus nel genere si vedano Stecher, op. cit. p. 48-58 e Vérilhac, op. cit., vol. I, n. 63-68. Si segnalano in particolare l'epigramma per Kleonikos di Mileto campione olimpico nella lotta a lui dedicato dal fratello (Ebert 65, Mileto, III a. C.), sul tema delle speranze di gloria del padre e della patria infrante da una morte improvvisa, gli epigrammi per il diciassettenne A $\gamma \alpha \dot{\theta} \alpha \nu \delta \varrho o \varsigma$, Peek, GV 1026 (Tomis, Scythia Minor, II d. C.) e per $\Delta \omega \mathrm{Q} \tilde{\alpha} \varsigma$, GV 1969 (Perinthos-Herakleia, Tracia, I/II d. C.), l'estrema scelta "la vittoria o la morte" è al centro di un breve epitaffio per il pugile A $\gamma \alpha \theta$ ós $\Delta \alpha$ í $\mu \omega v$ detto Ká $\mu \eta \lambda$ os (Cammello) 
temi e gli accenti dell'elogio atletico integrandoli ai motivi tipici del genere funerario, sino a far scattare l'inevitabile parodia. La satira a cui il poeta Lucillio diede sapientemente voce verso la metà del I sec. d. C., e che confluì negli epigrammi dell'XI libro dell'Antologia, ci conduce attraverso una galleria di antiatleti tumefatti e ci spalanca un repertorio di perfetti 'controelogi' che si possono gustare appieno solo se letti in parallelo alla documentazione epigrafica. Come ben sottolinea Louis Robert nella sua splendida analisi di questi componimenti ${ }^{32}$, l'arte di Lucillio si basa su una profonda conoscenza delle gare, del vocabolario tecnico e delle tematiche dell'elogio. Rispetto al topos del pubblico riconoscimento tributato all'atleta, che ben appare anche nel distico per il nostro giovane pugile tessalo, tutta l'efficacia del rovesciamento luciliano si apprezzerà nei versi che seguono (AP XI 77) :

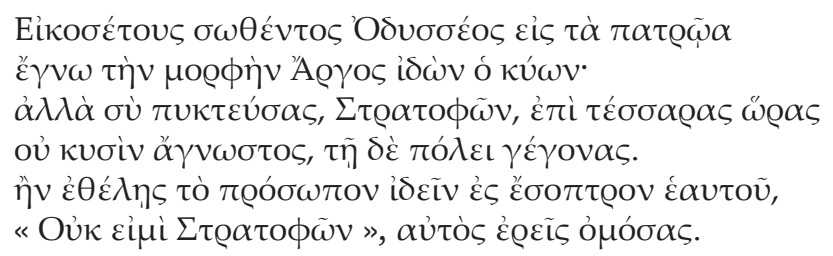

Quando dopo vent'anni Ulisse ritornò sano e salvo in patria, fu il suo cane Argo a riconoscerne le sembianze non appena lo vide. Tu invece, Stratofonte, sei diventato irriconoscibile non per i cani, ma per la città dopo aver fatto a pugni per (sole) quattro ore.

Se volessi guardare il tuo viso in uno specchio, tu stesso affermeresti sotto giuramento : "Non sono Stratofonte".

Eleonora SANTIN*
Collegium de Lyon, Lyon
Athanasios TzIAFALIAS
Larissa

di trentacinque anni giunto da Alessandria d'Egitto a Olimpia nella speranza, chiaramente vana, di guadagnare una vittoria da tempo sognata, vd. G.J. Te Riele, Inscriptions conservées au musée d'Olympie, BCH 88 (1964), p. 186-187 e BCH 89 (1965), p. 585-586, cf. Robert, cit. infra, p. 198201 e p. 288.

32. Cf. L. Robert, Les épigrammes satiriques de Lucillius sur les athlètes : parodie et réalités, in L'épigramme grecque (Entr. Fond. Hardt, XIV) Vandœuvres-Genève, 1968, p. 179-292.

* Ringrazio Lucia Floridi per i suoi preziosi suggerimenti riguardo alla non facile resa dell'epigramma di Lucillio. 


\section{SOMMAIRE}

Annie BÉLIS

Une inscription dépourvue de sens sur une amphore de Berlin?

Marie-Thérèse Cam et Yvonne Poulle-Drieux

Trilli, le bas-ventre du cheval (Végèce, mulom. 3, 4)

Nicole Guilleux

Strepsiade, Phidon, Phidippide : choix onomastiques et stratégie dramaturgique dans les Nuées

Nicole LANÉRÈs

Irènes et Tritirènes, réflexions sur la vocabulaire laconien de l'éphébie..

Claire Le Feuvre

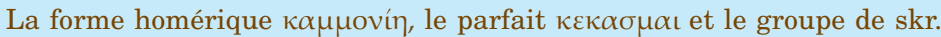
śámsati « louer »

Léopold MigeotTe

Les ressources financières des cités et des sanctuaires grecs : questions de terminologie et de classement.

Christian Nicolas

De l'étymologie pour l'œil à l'étymologie pour l'oreille : l'exemple de la prosthèse vocalique dans les Étymologies d'Isidore de Séville

Sylvie PitTia

Les méfaits de la paix (Denys d'Halicarnasse, Antiquités romaines 12,6,2)

Eleonora SANTin et Athanasios TZIAFALIAS

Un pugile ai piedi dell'Olimpo : un nuovo epigramma da Azoros (Tessaglia)

Joëlle SoLER

Lucius, parent de Plutarque, ou : comment lire les Métamorphoses d'Apulée

Gianluca Ventrella

Per l'attribuzione a Saturnino Secondo Salustio della ' $\Upsilon \pi o ́ \theta \varepsilon \sigma \iota \varsigma$ metrica dell'Edipo a Colono

Notes et Discussions : Antoine Pietrobelli - Hypothèses pour une génétique des plus anciens traités de la Collection hippocratique.

CHRONIQUe D'ÉTYMOLOGIE LATINE, $\mathrm{n}^{\circ} 4$

BULLETIN BIBLIOGRAPHIQUE

OUvRAGES REÇUS

RÉSUMÉS / ABSTRACTS 